\title{
Eliminating Mitochondrial DNA Competition for Nuclear DNA Primers
}

\author{
Steve Zullo, ${ }^{1,2}$ James L. Kennedy, ${ }^{2,6}$ Joel Gelernter, ${ }^{3,5}$ Michael H. Polymeropoulos, ${ }^{1}$ \\ Giovanni Tallini, ${ }^{4,8}$ Andrew J. Pakstis, ${ }^{2}$ Marvin B. Shapiro, ${ }^{7}$ Carl R. Merril, ${ }^{1}$ and Kenneth K. Kidd ${ }^{2}$
}

\begin{abstract}
${ }^{1}$ Laboratory of Biochemical Genetics, National Institute of Mental Health Neuroscience Center at St. Elizabeths, Washington, D.C. 20032; Departments of ${ }^{2}$ Genetics, ${ }^{3}$ Psychiatry, and ${ }^{4}$ Pathology, Yale University School of Medicine, New Haven, Connecticut 06510; ${ }^{5}$ West Haven Department of Veterans Affairs Medical Center, West Haven, Connecticut 06524; ${ }^{6} \mathrm{Clarke}$ Institute, University of Toronto, Canada M5T1R8; ${ }^{7}$ Division of Computer Research and Technology, National Institutes of Health, Bethesda, Maryland 20892
\end{abstract}

\begin{abstract}
Mitochondrial DNA (mtDNA) sequences were synthesized with nuclear DNA (nucDNA) sequencetagged site (STS) primers by mismatch priming in three independent studies of the human nuclear genome. Mismatch primer binding sites on the mtDNA were identified with from 6- to 10-bp identity at the 3 ' ends of the primers. In two of three cases, single-stranded mtDNA copies were gel-isolated with intended nuCDNA PCR products. During routine screening of the STSs, the radiolabeled gel-isolated products hybridized to polymorphic mtDNA restriction fragments. Intense signals after overnight exposure of radiolabeled PCR probes on Southern blots suggest contaminating mtDNA PCR products. The theoretical annealing temperatures of the mismatches were well below the annealing temperatures of the PCR primers, demonstrating annealing reactions driven by the molar surplus of the primers, that is, mass action. The probability that two primers (either one of a pair or both), designed to amplify nucDNA, will bind to and amplify mtDNA may be as high as 1 in 64, assuming that an identical match with only the $3^{\prime}$ hexanucleotide is sufficient for amplification. To circumvent this problem we have developed OLIGFIND, a program that has
\end{abstract}

This paper was presented in part in October 1991 as a poster at the Eighth International Congress on Human Genetics, Washington, D.C. ${ }^{8}$ Present address: Department of Pathology, Memorial Sloan Kettering Cancer Center, New York, New York 10021. identified the 104 of 4096 possible hexamers that are not present in human mtDNA. Our results suggest that time could be saved by designing STS primers with one of these 104 hexamers at the $3^{\prime}$ end. OLIGFIND can also evaluate primer $3^{\prime}$ ends for potential PCR products from mtDNA.

\section{S} (STSs) ${ }^{(1)}$ and $\mathrm{PCR}^{(2)}$ represent effective tools to help map and eventually sequence the human genome. Since the concept of STSs was proposed, several thousand STSs have been developed. ${ }^{(3)}$ An important step in the establishment of a STS is the selection of specific primer sequences to delineate the STS and reduce the possibility of a nonspecific PCR resulting from mismatch priming.

Sommer and Tautz ${ }^{(4)}$ found that binding of the 3 '-terminal trinucleotide of 17-mer primers was sufficient for weak amplification, and the binding of the 3'-terminal quadra- to octanucleotide resulted in good amplification. Their study was a qualitative attempt to answer a largely quantitative question. Nevertheless, they highlighted an important consideration to keep in mind when designing primers for PCR of specific nuclear DNA (nucDNA) sequences: if possible mismatch sequences are more abundant than the intended target sequence, amplification of the DNA between mismatch sequences may be favored. Mismatch priming is a technique that is used effectively for site-directed mutagenesis. ${ }^{(5,6)}$
One possible source of mismatch sequences in studies of nucDNA STSs is mitochondrial DNA (mtDNA), which is present at $\sim 1000$ copies of mtDNA genes per copy of nucDNA genes per cell. ${ }^{(7)} \mathrm{Be}-$ cause isolated nucDNA is invariably contaminated with mtDNA, a mtDNA sequence complementing the 3 '-terminal nucleotides of a nucDNA STS primer may compete with the intended target.

Consideration of the mitochondrial genome as a competitor for nucDNA primers can reduce the time spent in correcting misleading linkage information. A few points are relevant to this concept: 1) mtDNA contains $55.6 \%$ AT, and STSs are preferably constructed within the generally AT-rich untranslated regions of genes; (2) mtDNA contains a number of perfect and imperfect direct and inverted repeats, making interference with single primers likely; (3) all of the 13 proteins encoded by the mtDNA are membrane proteins, and in the cases where coding sequences are used to construct STSs, interference with membrane protein STS primers is likely the result of possibly similar transmembrane motifs. In this paper we describe the synthesis/amplification of mtDNA sequences with nucDNA primers to two membrane proteins and with a primer from a region encoding hydrophobic amino acids of a cytosolic neuronal protein.

During an attempt to identify a STS for the synaptophysin gene, mtDNA sequences were amplified by mismatch priming and confirmed by Southern hybridization. Likewise, during identification of a STS for the dopamine $\beta$-hydroxylase (DBH) gene, ${ }^{(8)}$ mtDNA sequences 
were again amplified by mismatch priming and confirmed by Southern hybridization. Furthermore, a case of "crossspecies" mismatched amplification was encountered. Following the strategy of Bunzow et $a .^{(9)}$ who isolated the rat dopamine D2 receptor (DRD2) cDNA by using the receptor family member hamster $\beta_{2}$-adrenergic receptor gene as a hybridization probe, we employed the rat DRD2 cDNA sequence to search for the human homolog (Grandy et al. ${ }^{(10)}$ reported the cloning of this gene as we began this stage of the project). Oligonucleotide primers based on the rat DRD2 cDNA sequence were synthesized. One of these cross-species primers amplified a mtDNA sequence, which was confirmed with cloning and sequence analysis. These three cases demonstrate mismatched primer PCR of mtDNA and strongly suggest the utility of checking nucDNA STS primers against the mtDNA sequence.

As an aid in designing nucDNA STS primers to avoid competition with mtDNA, we developed the computer program OLIGFIND to analyze the oligonucleotide frequencies of the human mtDNA sequence. We have also used the primer analysis computer program AMPLIFY $^{(11)}$ to calculate primabilities and stabilities for the primer-mtDNA mismatch binding sites encountered in our work. The "primabilities" are determined by giving decreasing weight to matches from the primer's 3'-terminal nucleotide to the $5^{\prime}$ nucleotide. Stabilities are calculated by consideration of match run lengths. Although the derived values for primability and stability cannot be regarded as predictive, they are an attempt to quantify primer mismatches.

Other factors, such as ionic strength and composition, primer, and template concentrations, contribute to the mismatched primer phenomenon. Recent improvements in PCR protocols may also aid in avoiding mismatch PCR. This paper encourages gene mappers to give heed to mtDNA in their efforts to study nucDNA sequences.

\section{MATERIALS AND METHODS}

The $100-\mu l$ synaptophysin STS PCR contained $500 \mathrm{ng}(1.26 \mathrm{fM})$ of human genomic DNA, $200 \mu \mathrm{M}$ of each dNTP, 2.5 units of Taq polymerase (Perkin-Elmer Cetus) in $50 \mathrm{~mm} \mathrm{KCl}, 10 \mathrm{~mm}$ Tris- $\mathrm{HCl}$ (pH 8.4), $1.5 \mathrm{~mm} \mathrm{MgCl}_{2}, 0.1 \%$ (wt/vol) gelatin, and $65 \mathrm{ng}$ (100 pM) of each primer: SP1, 5'-CTA TTT ATC TGT CTG AGC CC-3' (bases 1044-1063); ${ }^{(12)}$ and SP2, 5'-GTT GGT TCT GTC CTC CTA TT$3^{\prime}$ (bases 2070-2051). ${ }^{(12)}$ The following temperature profile was used: $94^{\circ} \mathrm{C}$ for 1 $\min ; 58^{\circ} \mathrm{C}$ for $1 \mathrm{~min} ; 72^{\circ} \mathrm{C}$ for $2 \mathrm{~min}$, for 30 cycles.

The DBH STS PCR has been described by Gelernter et al. ${ }^{\left({ }^{8}\right)}$ Briefly, the PerkinElmer Cetus GeneAmp kit was used with the buffers supplied; primer DB1, 5'-TCC ACC CGT GGG CCC GA-3' (bases 770 754); ${ }^{(13)}$ and primer DB2, $5^{\prime}$-cgc ccg ccg cgc ccc cgc ccc gtc ccg ccg ccc ccg ccc CTG CAA AGA CAC AGT CAT-3' (40-base GC clamp plus bases $401-417) .{ }^{(13)}$ The following temperature profile was used: $94^{\circ} \mathrm{C}$ for $1 \mathrm{~min} ; 45^{\circ} \mathrm{C}$ for $1 \mathrm{~min} ; 72^{\circ} \mathrm{C}$ for $1 \mathrm{~min}$, for 30 cycles.

The DRD2 gene PCR used the PerkinElmer Cetus GeneAmp Kit with the buffers supplied and $500 \mathrm{ng}(1.26 \mathrm{fm})$ of human placental DNA and $50 \mathrm{pm}$ of the D27 primer, 5'-CTT GCG GAA CTC GAT GTT G-3' (bases 1221-1203). ${ }^{(9)}$ The following temperature profile was used: $92^{\circ} \mathrm{C}$ for $40 \mathrm{sec} ; 55^{\circ} \mathrm{C}$ for $30 \mathrm{sec} ; 72^{\circ} \mathrm{C}$ for $1.5 \mathrm{~min}$, for 30 cycles, with final extension at $72^{\circ} \mathrm{C}$ for $5 \mathrm{~min}$. Cloning in pUC18 followed standard protocols. The sequence of the cloned PCR product was determined by the dideoxy method. ${ }^{(14)}$

Restriction analysis of PCR products followed the manufacturer's recommendations. Analysis of 22 singly restricted genomic DNA digests (polyscreen filters) has been described. ${ }^{(15)}$ Normal human renal $\mathrm{mtDNA}$ was isolated by $\mathrm{CsCl}$ gradient centrifugation as described. ${ }^{(16)}$ Wisconsin GCG software, ${ }^{(17)}$ Intelligenetics software, and the programs AMPLIFY ${ }^{(11)}$ and OLIGFIND were used for computer analysis.

\section{RESULTS AND DISCUSSION \\ Southern Blot and Computer Analysis of PCR Products}

\section{Synaptophysin PCR Primers}

PCR with primers SP1 and SP2, intended to amplify sequences in the synaptophysin gene, produced a single fragment of the expected size (1026 bp; bases 1044-2070) ${ }^{(12)}$ from human genomic DNA. Restriction analysis of the gel-isolated product with BglII produced the fragments expected, 653 and $374 \mathrm{bp}$, when viewed in a $2 \%$ agarose gel with ethidium bromide and UV light.

Hybridization of the radiolabeled PCR mixture to polyscreen filters produced intense signals overnight in discrete bands (data not shown). Intense signals after an overnight incubation at $-70^{\circ} \mathrm{C}$ on a Southern blot of genomic DNA are usually not expected when searching for a single-copy gene or STS. Repetitive sequences might be responsible; however, smears but not single bands would be expected in that case. An apparent polymorphism demonstrated a pattern consistent with maternal inheritance (Fig. 1). The intense signals and maternal inheritance encouraged us to investigate possible mtDNA amplification. Comparison of the Southern blot autoradiograph of the radiolabeled PCR mixture with that of CsCl-banded human mtDNA hybridization to polyscreen filters confirmed the presence of $\mathrm{mtDNA}$ amplification products (Fig. 2). The 1-

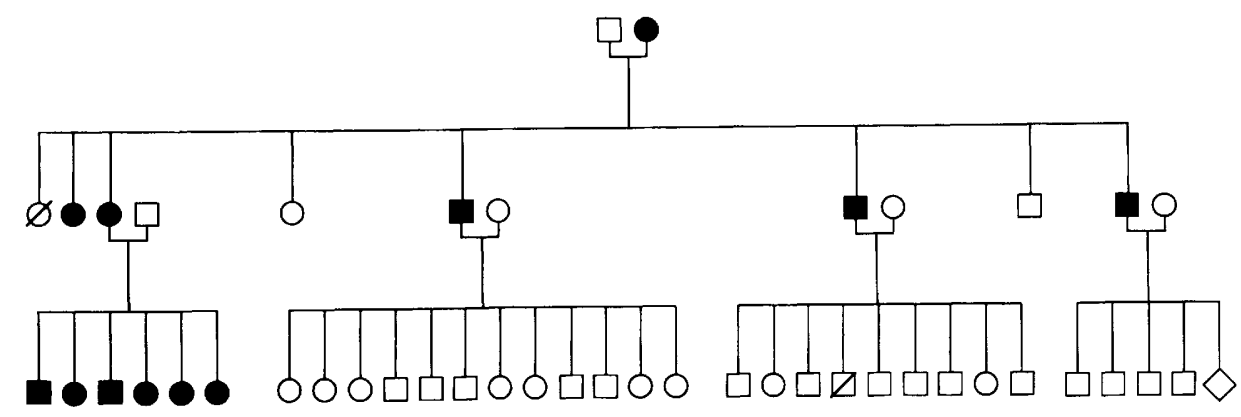

FIGURE 1 Pedigree of a mtDNA polymorphism detected by labeled PCR product with synaptophysin gene primers. This pedigree shows the segregation pattern of a polymorphism for MspI typed on a section of the Old Order Amish pedigree 110. ${ }^{(18)}$ Solid symbols represent those individuals missing the MspI site. Clearly, the segregation pattern is that of maternal inheritance, thus pointing to a mtDNA polymorphism detected by nucDNA primers. 


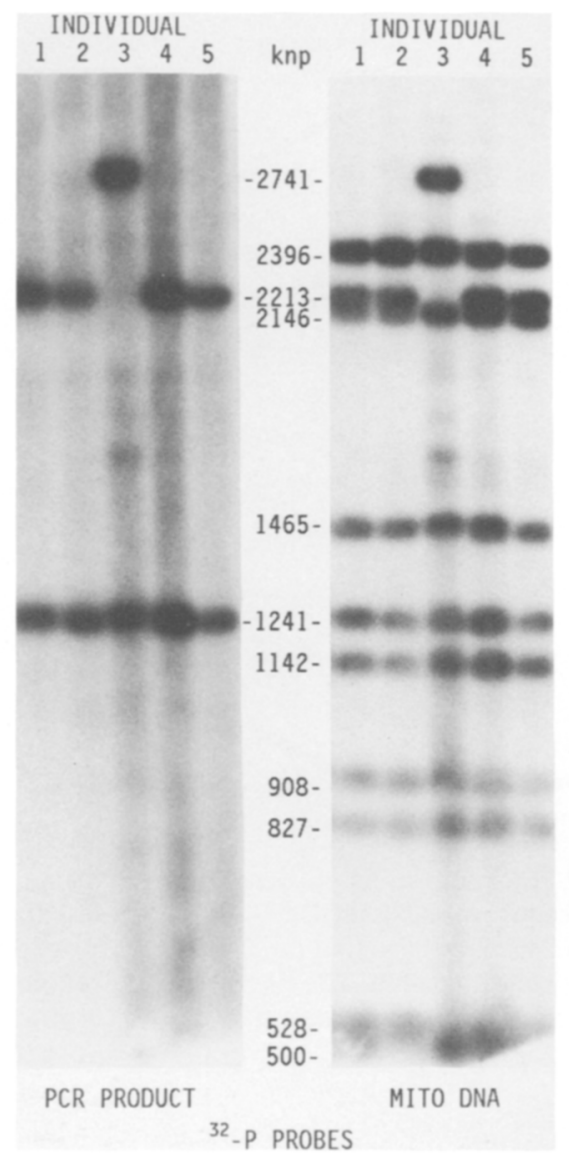

FICURE 2 Labeled PCR reaction detects mtDNA MspI restriction fragments in genomic DNA. (Left) MspI-digested human genomic DNA probed with unpurified, restriction digest-confirmed, labeled PCR product from a reaction with genomic primers for the synaptophysin gene. (Right) The same filter as at left, after removal of PCR probe, and reprobing with labeled, CsCl-banded human mtDNA. Genomic DNA from Old Order Amish pedigree $110 .^{(18)}$ Exposure durations: PCR Product, $12 \mathrm{hr}$; mtDNA, $45 \mathrm{~min}$.

kbp PCR product was gel-isolated, labeled, and hybridized to the polyscreen filters. The 2213- and 2741-bp mtDNA fragments, but not the 1241-bp mtDNA fragment, were hybridized (data not shown).

A WORDSEARCH analysis compared primers SP1 and SP2 with the human mtDNA sequence. ${ }^{(19)}$ The analysis revealed a number of possible priming sites on the mtDNA (Fig. 3a). All matches with at least $3^{\prime}$-hexamer identity were noted. Two sites, each with 3 '-octamer identity, could lead to a $1 \mathrm{kbp}$ PCR product (Table 1). A PCR product resulting from primers binding to these sites would hybridize to the 2213- and 2741- bp fragments revealed in Figure 2. In addition, a linearly amplified, singlestranded product from 14596L (or 14254L; see Fig. 3a) could be extended onto the 1241-bp MspI fragment (1212313364). MtDNA is not restricted by BglII, and $B g l I \mathrm{I}$ apparently completely restricted the gel-isolated 1-kbp PCR product. Because no uncut product was evident at the level of sensitivity of ethidium bromide fluorescence, the mtDNA products must have been in relatively low abundance compared with the nucDNA product. We believe that the mtDNA products were probably single-stranded polynucleotides resulting from binding of the SP2 primer to the site at $14596 \mathrm{~L}$. Gel isolation of the PCR product eliminated long single-stranded mtDNA products extending onto the 1241-bp MspI fragment.

\section{DBH PCR Primers}

PCR with the $\mathrm{DBH}$ primers generated a single fragment of the expected size (410 bp, 40 base GC clamp plus $370 \mathrm{bp}$, bases 401-770) ${ }^{(13)}$ from human genomic DNA. Restriction analysis of the gel-isolated product with $\mathrm{SacI}$ resulted in fragments of 325 and $85 \mathrm{bp}$, also as anticipated. However, hybridization of the gel-isolated 410-bp PCR product to polyscreen filters produced intense signals overnight, as well as a BanII RFLP (Fig. 4, left). In light of the data from the synaptophysin experiment, this finding alerted us again to possible mtDNA amplification. The mtDNA origin of the fragment hybridized by the PCR product was confirmed with labeled CsCl-banded human mtDNA (Fig. 4, right). MtDNA is not restricted by SacI, and SacI apparently completely restricted the gel-isolated 410-bp PCR product. Because no uncut product was evident at the level of sensitivity of ethidium bromide fluorescence, the mtDNA products must have been in relatively low abundance compared with the nucDNA product.

The primer sequences were also compared with the mtDNA sequence by WORDSEARCH. All matches with at least 3 '-hexamer identity were found (Fig. $3 b)$. A potential light-strand binding site at $4425 \mathrm{~L}$ (Table 1) could lead to a PCR product involving the restriction fragment from 3485 to 4431 . However, the only potential heavy-strand binding site downstream of this light-strand binding site is at $2407 \mathrm{H}$, which would yield a
mtDNA PCR product of $2 \mathrm{kbp}$, and thus is probably not recoverable with the gelisolated 410-bp PCR product. The results shown in Figure 4 were apparently the result of frequent binding of primer DB1 to the potential light-strand binding site at $4425 \mathrm{~L}$ and synthesis of variable-length polydeoxynucleotides, some of which were recovered along with the 410-bp PCR product during gel isolation.

\section{DRD2 PCR Primer}

Our search for the human DRD2 gene resulted in a 956-bp PCR product from human placental DNA with the D27 primer. Sequence analysis of the cloned PCR product revealed that the fragment included portions of the 16S rRNA gene and the NADH1 gene of human mtDNA. The primer matches the mtDNA sequence ${ }^{(19)}$ at both ends of the amplified fragment, the 3 '-terminal decamer at $2995 \mathrm{H}$, and the $3^{\prime}$-terminal nonamer at 3939L (Table 1). The cloned and sequenced fragment ostensibly contained the entire primer on both ends.

\section{MtDNA and Mismatch Priming}

Ruano et al. ${ }^{(23)}$ described PCR as a process with two components: (1) a target screening phase in which the desired DNA fragment is selected by primer binding, and (2) an amplification phase in which the desired DNA fragment copy number increases geometrically. A breakdown of the most important target screening phase of the PCR process is illustrated by continued mispriming of mtDNA. We have demonstrated that nucDNA primers can amplify mtDNA or synthesize single-stranded mtDNA by mismatch priming. Interestingly, sequence-confirmed geometric amplification occurred with a single primer, D27 (Table 1). Because genomic amplification with a single primer is gaining in popularity, ${ }^{(24,25)}$ it is prudent to minimize the possible binding of a nucDNA STS primer to mtDNA.

It has been noted that a disadvantage of PCR in analyzing transgenic organisms is the occasional false positive. ${ }^{(26)}$ MacQuitty suggested Southern blotting as an adjunct to identify false positives. However, caution is necessary. We have shown here that mtDNA in Southern blots can also create false positives. Autoradiographs that reveal hybridization in a short time, or demonstrate apparent 

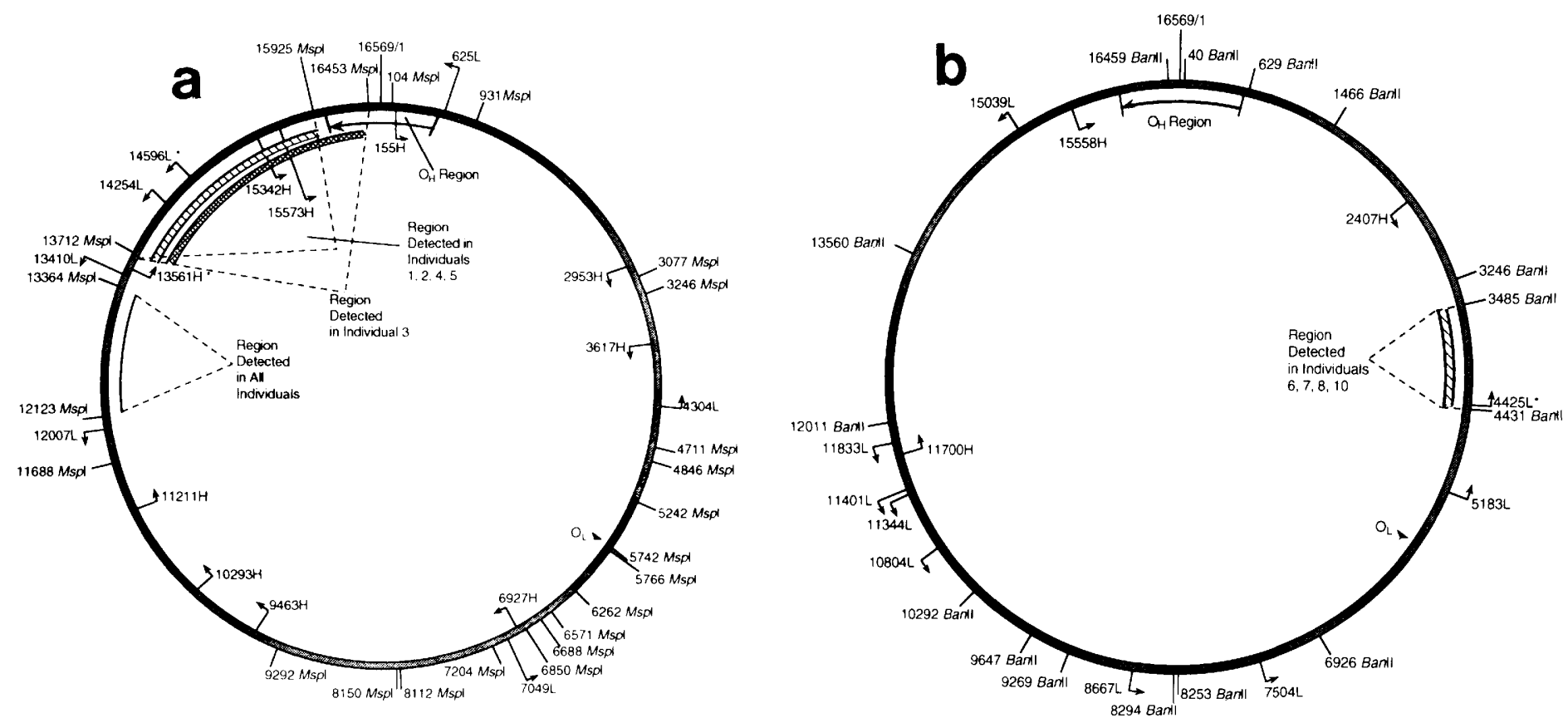

FIGURE 3 (a)Location of human mtDNA MspI restriction fragments hybridized by labeled aliquot of PCR reaction using synaptophysin primers, with possible priming sites indicated. The primer sequences were compared with the mtDNA sequence by WORDSEARCH.(17) All matches with at least 3'-hexamer identity are indicated; numbering refers to the 3' end of the putative binding site. (b) Location of human mtDNA BanII restriction fragment hybridized by labeled gel-isolated PCR product using DBH primers, with possible priming sites indicated. The primer sequences were compared with the mtDNA sequence by WORDSEARCH. ${ }^{(17)}$ All matches with at least $3^{\prime}$-hexamer identity are indicated; numbering refers to the $3^{\prime}$ end of the putative binding site.

maternal inheritance, should be noted and the possibility of mtDNA amplification ruled out. Because the concentration of contaminating mtDNA is high in a genomic DNA isolation (unless the nucDNA is purified further by $\mathrm{CsCl}$ banding), there can be enough singlestranded mtDNA sequences synthesized to hybridize to and reveal the mtDNA fragments on a blot. The mtDNA-derived products will probably not show up on an ethidium bromide gel check of the PCR reaction and may be recovered with an expected PCR product during gel isolation, as in the DBH experiment. Some single-stranded mtDNA products can also be eliminated during the gel isolation of a PCR product, as in the synaptophysin experiment.

It is particularly cogent to note that the annealing/melting temperatures of the primer-mtDNA mismatches identified in this paper are well below the annealing temperatures used in the PCRs (Table 1). Given the large molar surplus of primers used in PCR, kinetics strongly favor primer binding to mtDNA template, in spite of the annealing temperatures used during PCR. Thus, mtDNA offers an effective competitor of the prim-
er-to-nucDNA or primer-to-new strand binding reaction.

\section{Mathematics of NuCDNA Primer PCR of MIDNA and the Program OLIGFIND}

Primers constructed for nucDNA STS PCR should be compared with the mtDNA sequence to ensure that the $3^{\prime}$ terminus is not complementary to a mtDNA sequence. Single pentamer primers are used to create amplification fingerprints of soybeans. ${ }^{(27)}$ In the cases described above, 3'-octamer complementarity resulted in mismatch priming of human mtDNA. A reasonable number of possibilities of nonbinding oligomers on mtDNA can be constructed from the $4096\left(4^{6}\right)$ possible hexamers of A, C, G, and $\mathrm{T}$.

Of these 4096 hexamers, 104 are not found in mtDNA (Table 2), that is, 3992 do occur. With 33,138 nucleotides (16,569 per strand) in human mtDNA, the average number of times any one hexamer occurs in mtDNA is about eight (33138/3992). If we judge that the terminal $3^{\prime}$-hexamer binding is sufficient for primer-directed synthesis, the probability that any two nucDNA STS primers will bind to mtDNA in a fashion leading to PCR is $1 / 64$. That is, $(0.97)(1 / 2)(1 / 2)$ $(1 / 16)=0.97 / 2^{6}=0.97 / 64 \sim 1 / 64$, where 0.97 is the probability of a primer binding mtDNA (3992/4096), $1 / 2$ is the probability of second primer binding to opposite strand, $1 / 2$ is the probability of binding downstream of the first primer, and $1 / 16$ is the probability of the second primer binding the opposite strand within 1000 nucleotides of the first primer (an arbitrary separation limit for an efficient PCR reaction). However, as shown in this paper, geometric amplification is not necessary for competition by mtDNA for nucDNA STS primers to yield spurious results. Single-stranded synthesis of mtDNA can also confound the gene mapper. Theoretically, more mtDNA product than nucDNA product will be produced if $<14$ PCR cycles are run. The following four assumptions are made in this case:

1. Primer binds equally to mtDNA and nucDNA templates.

2. Both nucDNA homologs are bound.

3. MtDNA template is not bound in PCR orientation. 
TABLE 1 Match Characteristics of MtDNA Mismatch Priming Sites

\begin{tabular}{|c|c|c|c|c|c|}
\hline Primer & Match & $\begin{array}{l}\text { Percent } \\
\text { primability }^{\mathrm{a}}\end{array}$ & $\begin{array}{l}\text { Percent } \\
\text { stability }^{\mathrm{a}}\end{array}$ & $T_{\mathrm{m}}^{\mathrm{b}}$ & PCR $T_{\mathrm{m}}$ \\
\hline SP1 & $5^{\prime}$-CTATTTATCTGTCTGAGCCC - 3' & 80 & 43 & 32 & 58 \\
\hline mtDNA & $\begin{array}{l}3^{\prime}-\text { T ATGTGT T TGCGGACT CGGG - 5' } \\
13542^{\mathrm{c}} \\
13561\end{array}$ & & & & \\
\hline mtDNA & $\begin{array}{l}14596 \\
5^{\prime} \text { - AAT AGGAGAAGGCT TAGAAG - 3' }\end{array}$ & 78 & 33 & 28 & 58 \\
\hline SP2 & 3' - TTATCCTCCTGTCT TGGTTG - 5' & & & & \\
\hline mtDNA & $\begin{array}{l}4425 \\
5^{\prime} \text { - TCGGGCCCATACCCCGA - } 3 \text { ' }\end{array}$ & 81 & 40 & 38 & 45 \\
\hline DB1 & 3' - AGCCCGGGTGCCCACCT - 5' & & & & \\
\hline $\mathrm{D} 27$ & 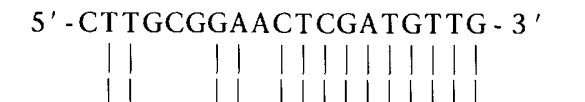 & 91 & 50 & 40 & 55 \\
\hline mtDNA & $\begin{array}{l}3^{\prime} \text { - CAAATGCTGGAGCT ACAAC - 5' } \\
2977 \quad 2995\end{array}$ & & & & \\
\hline mtDNA & $\begin{array}{l}3959 \\
3939 \\
5^{\prime} \text { - CAACATCGAATACGCCGCAGG-3' } \\
\end{array}$ & 86 & 41 & 50 & 55 \\
\hline D27 & 3' - GT TGT AGCTCA . . AGGCGTTC-5' & & & & \\
\hline
\end{tabular}

${ }^{a}$ Calculated by AMPLIFY.

${ }^{\mathrm{b}} \mathrm{AT}$ match $=2^{\circ} \mathrm{C}$; GC match $=4^{\circ} \mathrm{C}$.

'Numbering from Ref. 19.

4. MtDNA single gene copy per cell/ nucDNA single gene copy per cell $=10^{3}$. If mtDNA products accumulate as $n$, where $n=$ number of cycles, and nucDNA products accumulate as $2^{n}$, then mtDNA product/nucDNA product is $1000 n: 2^{n}$. At $n=13$, this ratio is $13,000: 8192$ and at $n=14$ it is $14,000: 16,384$. Therefore, mtDNA product $>$ nucDNA product for $n<14$.

Theoretically, in a normal 30-round PCR, $2^{30} \sim 1\left(10^{9}\right)$ copies of nucDNA products per cell are produced, whereas 30,000 , or $3\left(10^{4}\right)$, copies of singlestranded mtDNA products per cell are produced; therefore, mtDNA copy/ nucDNA copy $\sim 3\left(10^{-5}\right)$. The mtDNA/ nucDNA single gene copy ratio on the blot is presumably the same as in the PCR template sample, or $10^{3}: 1$. At a 1000 -fold abundance over nucDNA single copy gene sequences, hybridization to mtDNA sequences can create strong synaptophysin search, where singlestranded mtDNA products hybridized to the mtDNA fragments on the blot, in spite of being reduced in abundance by gel isolation of a discrete doublestranded fragment. Intense signals resulted after only $12 \mathrm{hr}$ of exposure.

To reduce the probability of competition by mtDNA for nucDNA STS primers, we developed the computer program OLIGFIND, which is used to find the exact occurrence of $n$-mers in a given sequence, where $n$ is chosen by the user to be between 5 and 10 . The program's output contains a count of the number of $n$-mers not occurring in the input strand and in the complementary strand. This is followed by a list of locations of those $n$-mers occurring one time in one strand and not in the other-two times, three times, and so on, up to the number of times specified.

In addition, OLIGFIND allows the user to specify a list of primers that are then tested for the possibility of producing PCR products from the given input signals. This was seen especially in the sequence. A good primer is one that occurs exactly once in each strand of the sequence, and with the two locations being within a specified number of base pairs.

The utility of this program lies in the simplicity and usefulness with which it alerts the user to possible PCR products and single-stranded extension products from mtDNA that can yield misleading results. Some of the newer techniques for enhancing specificity of PCR, for example, preamplification heating ${ }^{(28)}$ and heat-soak PCR, ${ }^{(29)}$ may also be helpful in preventing the potential difficulties resulting from mtDNA contamination of genomic DNA isolates.

OLIGFIND runs on PC computers and

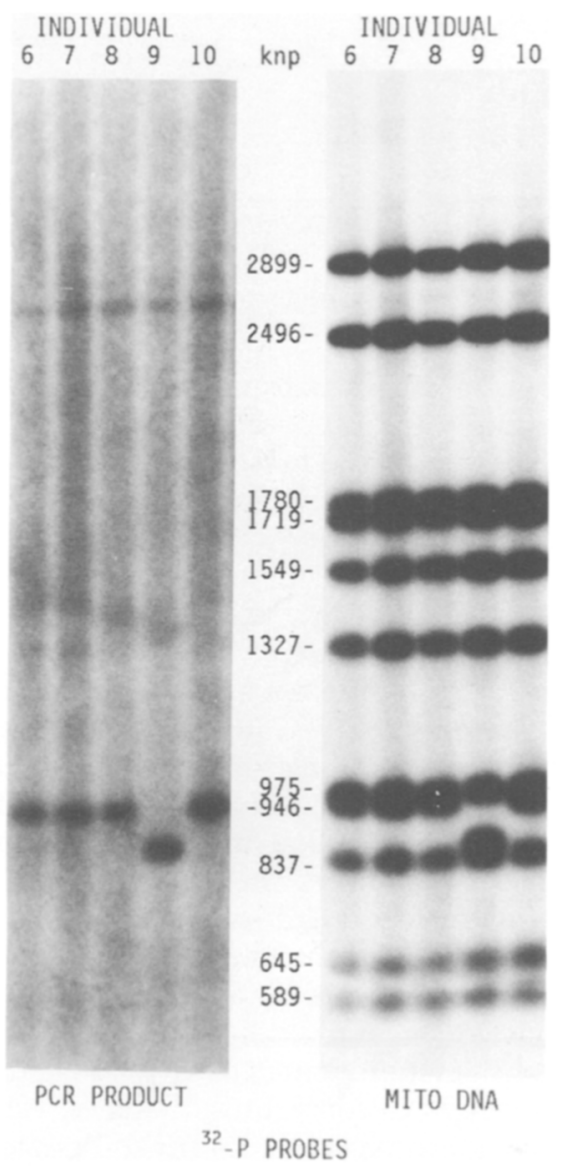

FIGURE 4 Labeled gel-isolated 410-bp PCR product detects mtDNA BanII restriction fragments in genomic DNA. (Left) BanlI-digested human genomic DNA probed with labeled, gel-isolated PCR product, from a reaction with genomic primers for the DBH gene. (Right) The same filter as at left, after removal of PCR probe, and reprobing with labeled, $\mathrm{CsCl}$ banded human mtDNA. Shown are genomic DNA of individual $6,^{(20)}$ individuals 7 and $10,{ }^{(21)}$ individuals 8 and 9. ${ }^{(22)}$ Exposure durations: PCR product, $12 \mathrm{hr}$; mtDNA, $45 \mathrm{~min}$. 
TABLE 2 Hexamers Not Found in Human MtDNA

\begin{tabular}{|c|c|c|c|c|c|c|c|c|c|}
\hline No. & Hexamer & No. & Hexamer & No. & Hexamer & No. & Hexamer & No. & Hexamer \\
\hline 103 & AACGCG & 142 & AAGATC & 190 & AAGT TC & 282 & ACACGC & 283 & ACACGG \\
\hline 364 & ACCGGT & 391 & ACGACG & 412 & ACGCGT & 436 & ACGTAT & 442 & ACGTGC \\
\hline 566 & AGATCC & 567 & AGATCG & 568 & AGATCT & 610 & AGCGAC & 621 & AGCGT A \\
\hline 796 & ATACGT & 1130 & CACGGC & 1135 & CACGTG & 1321 & CCAGGA & 1387 & CCCGGG \\
\hline 1433 & CCGCGA & 1434 & CCGCGC & 1468 & CCGTGT & 1561 & CGACGA & 1562 & CGACGC \\
\hline 1571 & CGAGAG & 1575 & CGAGCG & 1591 & CGATCG & 1592 & CGATCT & 1633 & CGCGAA \\
\hline 1634 & CGCGAC & 1635 & CGCGAG & 1637 & CGCGCA & 1639 & CGCGCG & 1641 & CGCGGA \\
\hline 1648 & CGCGTT & 1651 & CGCTAG & 1655 & CGCTCG & 1670 & CGGACC & 1682 & CGGCAC \\
\hline 1686 & CGGCCC & 1698 & CGGGAC & 1718 & CGGTCC & 1734 & CGTACC & 1753 & CGTCGA \\
\hline 1754 & CGTCGC & 1756 & CGTCGT & 1762 & CGTGAC & 1770 & CGTGGC & 1786 & CGTTGC \\
\hline 1831 & CTAGCG & 1890 & CTCGAC & 1895 & CTCGCG & 1911 & CTCTCG & 1954 & CTGGAC \\
\hline 2080 & GAACTT & 2146 & GACGAC & 2202 & GAGCGC & 2265 & GATCGA & 2266 & GATCGC \\
\hline 2272 & GATCTT & 2311 & GCAACG & 2332 & GCACGT & 2375 & GCCACG & 2415 & GCCGTG \\
\hline 2439 & GCGACG & 2446 & GCGATC & 2458 & GCGCGC & 2459 & GCGCGG & 2462 & GCGCTC \\
\hline 2487 & GCGTCG & 2492 & GCGTGT & 2583 & GGACCG & 2616 & GGATCT & 2711 & GGGCCG \\
\hline 2759 & GGTACG & 2775 & GGTCCG & 2887 & GTCACG & 2898 & GTCCAC & 2899 & GTCCAG \\
\hline 2903 & GTCCCG & 2914 & GTCGAC & 2915 & GTCGAG & 2919 & GTCGCG & 2920 & GTCGCT \\
\hline 2926 & GTCGTC & 2967 & GTGCCG & 2978 & GTGGAC & 3176 & TACGCT & 3337 & TCAAGA \\
\hline 3401 & TCCAGA & 3431 & TCCGCG & 3451 & TCCTGG & 3463 & TCGACG & 3470 & TCGATC \\
\hline 3481 & TCGCGA & 3483 & TCGCGG & 3511 & TCGTCG & 3561 & TCTGGA & 3577 & TCTTGA \\
\hline 3685 & TGCGCA & 3687 & TGCGCG & 3781 & TGTACA & 3943 & TTCGCG & & \\
\hline
\end{tabular}

Identified by the program OLIGFIND, which searches both strands of the human mitochondrial genome for each of the 4096 hexamers.

can be obtained from the authors via e-mail using anonymous FTP, with a binary transfer mode. The file to transfer is OLIGFIND.EXE, which resides on the National Institutes of Health Helix computer under the mail address mbs helix.nih.gov, and is found in the directory called mbs.

Although there are few rigorous programs or published studies that evaluate mismatch priming errors, the AMPLIFY program $^{(11)}$ is useful in guiding the worker in the design of primers. AMPLIFY determines the primability of a primer on a target sequence by giving decreasing weight to matches from the primer's $3^{\prime}$-terminal nucleotide to the $5^{\prime}$ nucleotide. Stabilities are calculated by consideration of match run lengths, that is, length of consecutive matches. Table 1 exhibits the range of primabilities and stabilities determined by AMPLIFY for the genomic primer-mtDNA mismatches realized in our studies. The AMPLIFY program assigns 59\% of the match weights to the 3 '-terminal hexamer.

These programs are not definitive devices to ensure the absence of mismatch priming nor are they predictive of mismatch priming. For instance, primer SP1, with $80 \%$ primability and $43 \%$ stability on the mtDNA template, appar- ently did not yield mismatch primed extension products, but primer SP2, with $78 \%$ primability and $33 \%$ stability, did (Table 1).

We have developed OLIGFIND to rapidly decipher potential genomic primermtDNA mismatches. Use of the hexamers not occurring in human mtDNA (Table 2) provides a strategy to quickly design primers for human STS construction with a minimal likelihood of mtDNA interference. As more STSs are developed for a genome, contaminating mtDNA may become more of a problem in defining new STSs and STS primers may become limited by the codon usage of the organism. Incorporating these 104 hexamers onto the $3^{\prime}$ end of human genomic STS primers will probably eliminate competition from human mtDNA.

\section{ACKNOWLEDGMENTS}

We thank J.M. Eisenstadt of the Department of Genetics at Yale University School of Medicine for use of his laboratory for parts of this investigation, and G.J. Creed, K. Payza, and D. Sabourin for insightful discussions of the final draft. This work was supported in part by funds from the U.S. Department of Vet- erans Affairs Medical Research Program (merit review to J.G.) and by National Institute of Mental Health grant MH00931 to J.G. Information about AMPLIFY can be obtained from W.R. Engels at the e-mail address wrengels@macc. wisc.edu.

\section{REFERENCES}

1. Olson, M., L. Hood, C. Cantor, and D. Botstein. 1989. A common language for physical mapping of the human genome. Science 245: 1434-1435.

2. Mullis, K.B. and F.A. Faloona. 1987. Specific synthesis of DNA in vitro via a polymerase-catalyzed chain reaction. Methods Enzymol. 155: 335-350.

3. Pearson, P.L., B. Maidak, M. Chipperfield, and R. Robbins. 1991. The human genome initiative-Do databases reflect current progress? Science 254: 214-215.

4. Sommer, R. and D. Tautz. 1989. Minimal homology for PCR primers. Nucleic Acids Res. 17: 6749 .

5. Helmsley, A., N. Arnheim, M.D. Toney, G. Cartopassi, and D.J. Galas. 1989. A simple method for site-directed mutagenesis using the polymerase chain reaction. $\mathrm{Nu}$ cleic Acids Res. 17: 6545-6551. (Corrigendum: Nucleic Acids Res. 17: 8915).

6. Kuipers, O.P., H.J. Boot, and W.M. deVos. 
1991. Improved site-directed mutagenesis method using PCR. Nucleic Acids Res. 19: 4558.

7. Clayton, D.A. 1982. Replication of animal mitochondrial DNA. Cell 28: 693-705.

8. Gelernter, J., P.V. Gejman, S. Bisighini, and K.K Kidd. 1991. Sequence tagged site (STS) TaqI RFLP at dopamine $\beta$-hydroxylase (DBH). Nucleic Acids Res. 19: 1957.

9. Bunzow, J.R., H.H.M. VanTol, D.K. Grandy, P. Albert, J. Salon, M. Christie, C.A. Machida, K.A. Neve, and O. Civelli. 1988. Cloning and expression of a rat D2 dopamine receptor cDNA. Nature 336: 783-787.

10. Grandy, D.K., M.A. Marchionni, H. Makam, R.E. Stofko, M. Alfano, L. Frothingham, J.B. Fischer, K.J. Burke-Howie, J.R. Bunzow, A.C. Server, and O. Civelli. 1989. Cloning of the cDNA and gene for a human $\mathrm{D}_{2}$ dopamine receptor. Proc. Natl. Acad. Sci. 86: 9762-9766.

11. Engels, W. Personal communication.

12. Sudhof, T.C., F. Lootspeich, P. Greengard, E. Mehl, and R. Jahn. 1987. The cDNA and derived amino acid sequences for rat and human synaptophysin. Nucleic Acids Res. 15: 9607.

13. Kobayashi, K., Y. Kurosawa, K. Fujita, and T. Nagatsu. 1989. Human dopamine $\beta$-hydroxylase gene: Two mRNA types having different 3 '-terminal regions are produced through alternative polyadenylation. Nucleic Acids Res. 17: 1089-1102.

14. Sanger, F., S. Nicklen, and A.R. Coulson. 1977. DNA sequencing with chain-terminating inhibitors. Proc. Natl. Acad. Sci. 74: 5463-5467.

15. Kidd, J.R., Y. Matsubara, C.M. Castiglione, K. Tanaka, and K.K. Kidd. 1990. The locus for the medium-chain acyl-CoA dehydrogenase gene on chromosome 1 is highly polymorphic. Genomics 6: 89-93.

16. Hadler, H.I., B. Dimitrijevic, and R. Mahalingam. 1983. Mitochondrial DNA and nuclear DNA from normal rat liver have a common sequence. Proc. Natl. Acad. Sci. 80: 6495-6499.

17. Devereux, J., P. Haeberli and O. Smithies. 1984. A comprehensive set of sequence analysis programs for the VAX. Nucleic Acids Res. 12: 387-395.

18. Egeland, J.A., D.S. Gerhard, D.L. Pauls, J.N. Sussex, K.K. Kidd, C.R. Allen, A.M. Hostetter and D.E. Housman. 1987. Bipolar affective disorders linked to DNA markers on chromosome 11. Nature 325: 783-787.

19. Anderson, S., A.T. Bankier, B.G. Barrell, M.H.L. deBruijn, A.R. Coulson, J. Drouin, I.C. Eperon, D.P. Nierlich, B.A. Roe, F. Sanger, P.H. Scheier, A.J.H. Smith, R. Staden, and I.G. Young. 1981. Sequence and organization of the human mitochondrial genome. Nature 290: 457-465.

20. Pauls, D.L., A.J. Pakstis, R. Kurlan, K.K. Kidd, J.F. Leckman, D.J. Cohen, J.R. Kidd,
P. Como, and R. Sparkes. 1990. Segregation and linkage analyses of TS. $J$. Am. Acad. Child Adolescent Psychiatr. 29: 195203.

21. Kurlan, R., J. Behr, L. Medved, I. Shoulson, D.L. Pauls, J.R. Kidd, and K.K. Kidd. 1986. Familial Tourette's syndrome: Report of a large pedigree and potential for linkage analysis. Neurology 36: 772-776.

22. Kidd, J.R., C.M. Castiglione, K.E. Davies, A.J. Pakstis, J. Gusella, R.S. Sparkes, P. Pearson, H. Willard, and K.K. Kidd. 1985. Mapping the locus for X-linked ocular albinism. Am. J. Hum. Genet. (Suppl.) 37: A161.

23. Ruano, G., D.E. Brash and K.K. Kidd. 1991. PCR: The first few cycles. Amplifications. Forum PCR Users 7: 1-4.

24. Welsh, J. and M. McClelland. 1990. Fingerprinting genomes using PCR with arbitrary primers. Nucleic Acids Res. 18: 7213-7218.

25. Williams, J.G.K., A.R. Kubelik, K.J. Livak, J.A. Rafalski, and S.V. Tingey. 1990. DNA polymorphisms amplified by arbitrary primers are useful as genetic markers. $\mathrm{Nu}$ cleic Acids Res. 18: 6531-6535.

26. MacQuitty, J. 1992. vide Fox, S. PCR increasingly employed in agricultural and veterinary biotechnology. Genet. Eng. News 12(9): 6-9.

27. Gresshoff, P. 1992. vide Fox, S. PCR increasingly employed in agricultural and veterinary biotechnology. Genet. Eng. News 12(9): 6-9.

28. D'Aquila, R.T., L.J. Bechtel, J.A. Videler, J.J. Eron, P. Gorczyca, and J.C. Kaplan. 1991. Maximizing sensitivity and specificity of PCR by preamplification heating. Nucleic Acids Res. 19: 3749.

29. Ruano, G., E.M. Pagliaro, T.R. Schwartz, K. Lamy, D. Messina, R.E. Gaensslen, and H.C. Lee. 1992. Heat-soaked PCR: An efficient method for DNA amplification with applications to forensic analysis. BioTechniques 13: 266-274.

Received January 22, 1993; accepted in revised form June 3, 1993. 


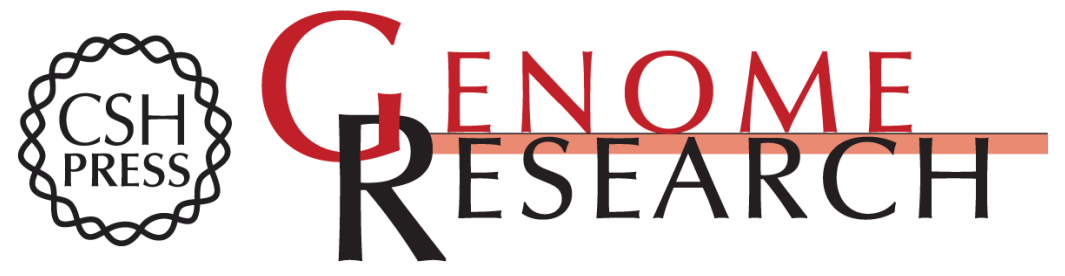

\section{Eliminating mitochondrial DNA competition for nuclear DNA primers.}

S Zullo, J L Kennedy, J Gelernter, et al.

Genome Res. 1993 3: 39-45

References This article cites 28 articles, 6 of which can be accessed free at: http://genome.cshlp.org/content/3/1/39.full.html\#ref-list-1

License

Email Alerting

Receive free email alerts when new articles cite this article - sign up in the box at the Service top right corner of the article or click here.

\section{Affordable, Accurate Sequencing.}

To subscribe to Genome Research go to: https://genome.cshlp.org/subscriptions 